\title{
Automated Attendance Monitoring and Personal Intelligent system
}

\author{
Aniket Shah ${ }^{1}$, Amruta Tuptewar ${ }^{2}$, Pradnya Yeole ${ }^{3}$, Prof.L.J.Sankpal ${ }^{4}$ \\ BE Student, Department of Computer Engineering, Sinhgad Academy of Engineering, Pune, India ${ }^{1,2,3}$ \\ Professor, Department of Computer Engineering, Singhad Academy of Engineering, Pune, India ${ }^{4}$
}

\begin{abstract}
In today's world, a paper based approach is followed for marking attendance, where the students sign on the attendance sheets. This data is then manually entered into the system. Managing the attendance of the students during lectures is a difficult task and it becomes more difficult during the report generation phase and manual computation produces errors and also wastes a lot of time. For this reason, the development of Automated Attendance Monitoring System (AMS) using android platform is proposed. Additionally, implementation of an intelligent system which will interact with users and provide environment where chatbot will interact to provide dedicated chat. So, user can access database using internet enabled Smartphone to solve his queries and get desired output with voice chat.
\end{abstract}

Keywords: Attendance Monitoring System (AMS), android platform, marking attendance on one click, user authentication, database, report generation, Interactive System.

\section{INTRODUCTION}

In almost every institution and organization, attendance monitoring is a very important process. The current method involves the use of sheets of paper or books in taking students attendance. This method could easily allow for impersonation and the attendance sheet could be lost or damaged. Taking attendance is thus time consuming using this traditional approach and hence there is a need of an automated and a reliable system.

The Attendance Monitoring System(AMS) will provide the needed solution. The system consists of two apk files, one for the teacher and one for the student respectively, which are installed on their android devices. The AMS will be used to mark the attendance of the students and will also be used to generate reports of all the students and thus will enable the faculty members to keep track of student's record. Rather than signing on the attendance sheets, the student will mark the attendance by just a single click on his device. Also, the teacher has the facility to generate reports on a single click. There is a facility to generate report of one or more than one student.

Chatbot uses natural language to facilitate turn by turn communication between user and computer. HumanComputer dialogue (chatbot) has been developed with interaction through text and speech which provide great flexibility and ease of interaction. While developing these type of systems we have to face many challenges like difference between human-human conversation and human-chatbot, what domains given to chat brain to make it more interactive, what technologies are selected etc. It draws human into conversation, using AIML (Artificial Intelligent Mark-up Language) a version of XML and it was released under the GNU GPL. AIML interpreters are launched under free or open source license.

\subsection{Related Works}

Different methods and principles have been applied to effectively monitor the attendance of the students. A system providing an improvised electronic card and card reader serially interfaced to the digital computer system was proposed, which is an embedded computer based attendance management system (Shoewu, O. O. M. Olaniyi, and Lawson et al, 2011)[6] A wireless attendance management system used the iris of the individual for authentication (Kadry, S. and M. Smaili et al, 2010)[4]. All processes like capturing the imageof iris recognition, extracting minutiae, storing and matching used an off-line iris recognition management system.[5]

1.2 AI Concepts

Artificial intelligence is a branch of computer science to study and develop intelligent machine. Different areas of artificial intelligence which considered are thinking machine, Expert System. Major domain considered in this project to perform above areas is Natural Language Processing. Basic aim is to see whether our intelligent system is strong enough to guide and explain all related input methods. Internet bot is the best choice to implement and study above domains. The System use is improved version with sophisticated natural language processing which give and take input by speech and text in general English

\section{SYSTEM OVERVIEW}

The proposed system provides solution to lecture attendance problems through the use of AMS that is interfaced to the server. The students and teacher will have their android devices. At first, the student enters his/ her details in the system through the application. The student also enters his/her parent's details. Every student is given a unique student id and they have their password. The student enters this Id and other personal details (Name, Department, Division, and Semester etc). The student then log's on into his account.The teacher, on his/her device, fills the details. The faculty members too, are assigned unique faculty id. They too have to enter their details and all the data is recorded in the database. After this, the teacher, activates the application that is on the server and 
only when the application is active, the student can mark the attendance by one click. The teacher can then generate reports by a single click. The teacher will also have the access to the list of students attending the lecture and can even modify the list if required.Reports can be generated as and when required. Facility is provided to generate weekly as well as monthlyreports. At the end of the month, SMS's can be sent to the parents/guardians of the students, thus informing them about their wards attendance.

\subsection{System Design}

The Attendance Monitoring System being a client-server approach, and follows a specific hardware and software architecture. The main challenge here is integrating both the hardware and software components to work together.

\subsubsection{Software Architecture}

The software architecture consists of: the database, the application program and the server.

\subsubsection{Database}

The database consists of a number of tables, which stores records implemented in phpmyadmin- MySQL. MySQL is easy, fast and efficient and can store a large number of records and requires a little configuration.

\subsubsection{Application Program}

The application program is developed with Android programming language using Eclipse framework. The application program provides user interface to both the faculty members as well as the students. Programming in Android is simple, user friendly and android offers an excellent data connectivity.

\subsubsection{Server}

The server is deployed on the personal computer using apache-Tomcat7. Tomcat7 is free, robust and easy to deploy.

\section{METHODOLOGY AND FLOWCHART}

Every user is authenticated based on his/her unique user identification number. This unique identification number is the number present on the ID cards of the faculty members and the students. Both the faculty members and the students register themselves by signing in into the system. A flag is set to a default value of false in the system. This flag can be altered only by the teachers and only when the flag is true, the students can mark their attendance. During the marking phase, the teacher of the concerned subject, activates the application by triggering it from his/her device and in turn changing the value of the flag to true. The students can now mark the attendance for that particular lecture by a single click. The teacher then deactivates the app after sometime (usually after a minute) by again changing the value to false. The teacher is given facility to generate reports as soon as the students are done marking their attendance. The teacher can mark the attendance of a particular student if at all any student cannot mark the attendance due to some technical issues. Similarly, the teacher can delete the attendance of the students. The next phase here is report generation. The code for generating reports is written using JSP. Only the admin, the concerned staff or faculty member is given the authority to access this page. He/she can search for a particular student in the database and can generate reports monthly or weekly. Also, periodic SMS is sent to the student's parents by the admin. This feature is enabled by connecting the system to an SMS gateway.

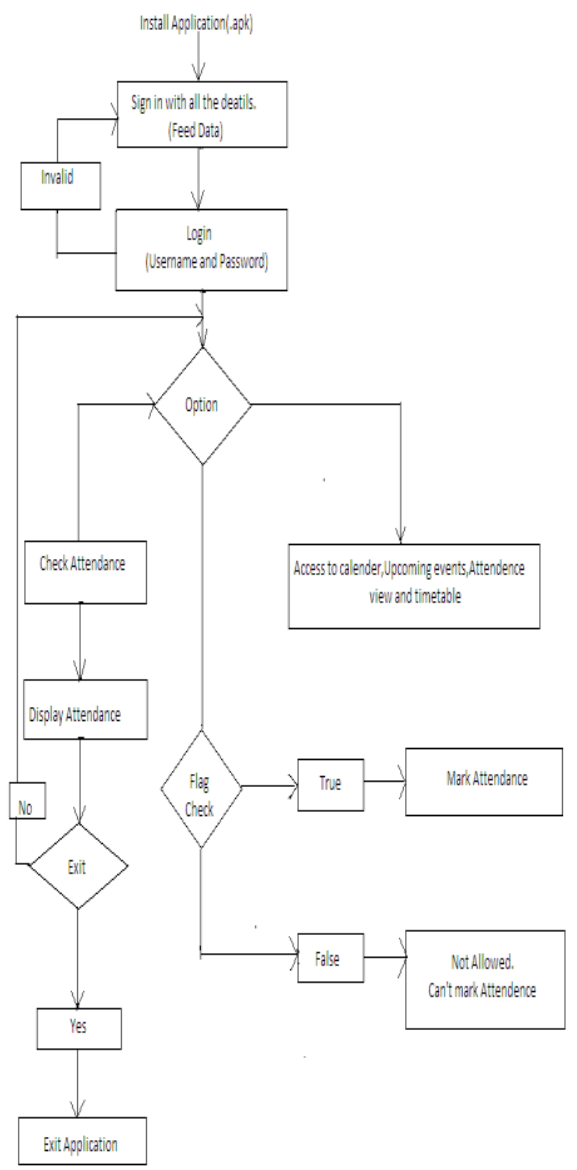

FIG.3

\section{PERSONAL ASSISTANT}

BOT lies on a web server so to access this application one needs internet enabled device such as computer, smartphone, tablet, pda etc.BOT uses 3-tier architecture. In 3-tier Architecture there exists 3 layers vies presentation tier, business tier and data tier. In BOT system web browser at the client side comes under presentation tier, input processing and pattern matching at the server side comes under business tier and AIML and .brn(Brain) file comes under data tier. Whenever user has a query to ask he just have to connect to the application through web browser using predefined web address.

When connected, one can start conversation with the bot. User can initiate general or technical talk with the bot. Bot accepts input either from keyboard or voice input and answers the query in the form of both voice and text. So here user gets lots of flexibility in obtaining answer for the given query. They can ask their query using voice input and get their answers back in voice form. When input is generated by client request is transferred to server. Server checks weather .brn file (also called as brain which contains all knowledge to resolve query) is present then load the file.Apply pattern matching algorithm and interpret user input and send the response back to client. 


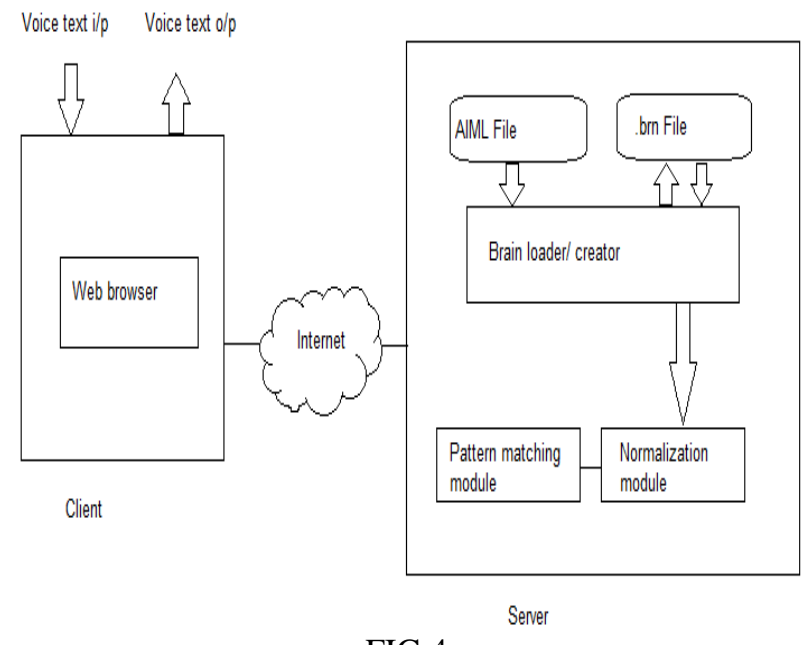

4.1 Real Applications

The system can be used as a dedicated system which gives information regarding students attendance when inputs are given as name or roll no. Also same system can be used as providing information regarding various subjects and general topics. .He can also enquire about few things related to college using voice. We are using some NLP logic over here to train the system as well as to get answers.

BOT $>$ hi

Hi what is my total attendance?

BOT $>69 \%$ til 2th November

How many more lectures should I attend to improve my attendance upto $75 \%$

BOT $>$ You need to attend 18 more lectures.

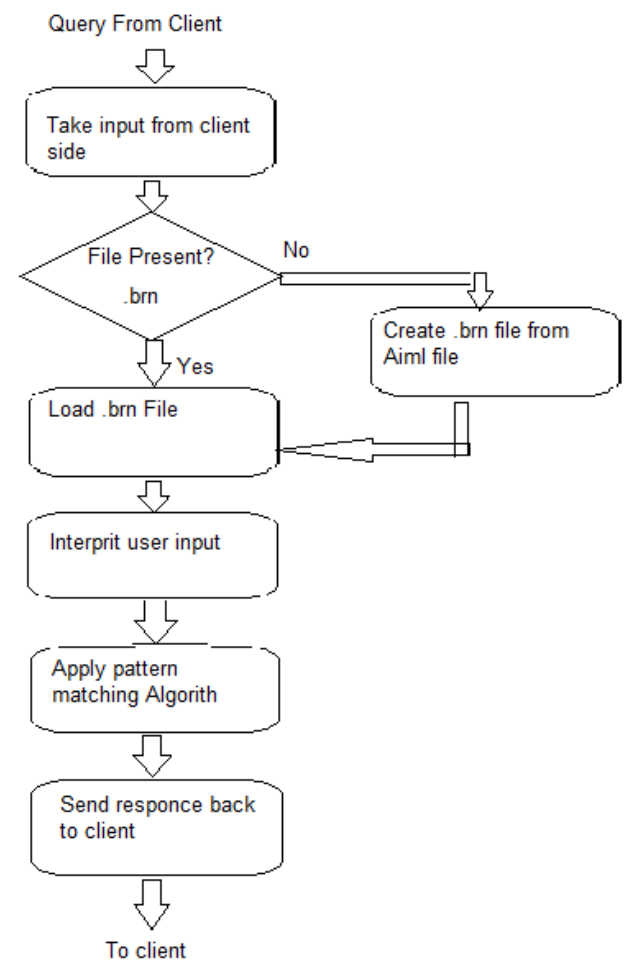

\section{CONCLUSIONS}

In this paper, we proposed a new system for monitoring attendance of the students using android platform. The results showed improvements in accuracy as compared to using user-based paper-based approach. Moreover the proposed technique provides an easy way for generating reports. .Student can also enquire about few things related to college using voice. We are using some NLP logic over here to train the system as well as to get answers. It uses simple pattern matching technique. It give flexibility to publish online. It have flexibility of speech reorganization input and output. We can provide as many input-output for general or specific domain.

\section{REFERENCES}

[1]. Shoewu, O. O. M. Olaniyi, and Lawson (2011), Embedded Computer-Based Lecture Attendance Management System,African Journal of Computing and ICT (Journal of IEEE Nigeria Computer Section), 4(3): $27-36$

[2]. Shoewu, O. and O.A. Idowu (2012), Development of Attendance Management System using Biometrics, Pacific Journal of Science and Technology, 13(1):300-307

[3]. Braun, A. (2003). Chatbots in der Kundenkommunikation (Chatbots in customer communication).Springer, Berlin

[4]. Steven Bird, Ewan Klein and Edward Loper (2009).Natural Language Processing byO'Reilly Media, Inc

[5]. International Journal of Applied Engineering Research, ISSN $0973-$ 4562, Vol. 8, No. 19 (2013)

[6]. International Journal of Current Engineering and Technology EISSN 2277 - 4106, P-ISSN 2347 - 51612014

FIG.4.1 